\title{
Formulation Design for Orally Disintegrating Tablets Containing Enteric-Coated Particles
}

\author{
Yutaka Okuda, ${ }^{* a}$ Yasunobu Okamoto, ${ }^{a}$ Yosuke Irisawa, ${ }^{b}$ Kazuto Okimoto, ${ }^{a}$ Takashi Osawa, ${ }^{a}$ and \\ Shinji Yamashita ${ }^{c}$ \\ ${ }^{a}$ Towa Pharmaceutical Co., Ltd.; 26-7 Ichiban-cho, Kadoma, Osaka 571-0033, Japan: ${ }^{b}$ Towa Pharmaceutical Co., \\ Ltd.; 34-2 Taiheidai, Shoo-cho, Katsuta-gun, Okayama 709-4321, Japan: and ${ }^{c}$ Faculty of Pharmaceutical Sciences, \\ Setsunan University; 45-1 Nagaotouge-cho, Hirakata, Osaka 573-0101, Japan. \\ Received September 26, 2013; accepted February 24, 2014
}

The purpose of this study was to investigate the applicability of our newly developed technology (RACTAB $^{\circledR}$ technology) for preparing orally disintegrating tablets (ODTs) containing enteric-coated particles. Tamsulosin hydrochloride (TAM) was used as a model drug contained in the enteric-coated particles. Enteric-coated particles containing TAM (ECP-T) were prepared by spray coating a mixture of TAM with controlled-release materials. ECP-T was then mixed with rapidly disintegrating granules (RDGs), which were prepared using the suspension spray-coating method, and was tableted to form ODTs $\left(\right.$ ODT $\left._{\mathrm{RAC}}\right)$. ODT $_{\text {RAC }}$ was evaluated for its hardness, thickness, internal structure (X-ray-CT scanning), functional properties (controlled-release profile), and in vivo disintegration time. Since RDGs with micronized ethylcellulose (MEC) increased tablet hardness by increasing the contact frequency between granules, ODT $_{\text {RAC }}$ containing ECP-T exhibited high hardness $(>50 \mathrm{~N})$ and low friability $(<\mathbf{0 . 5 \%})$ with a relatively low compression force. After tableting, the structure of ECP-T in ODT $_{\mathrm{RAC}}$ remained intact and no damage was observed on the surface. ECP-T recovered from ODT $_{\mathrm{RAC}}$ showed the same dissolution profile of TAM in Japanese Pharmacopoeia (JP) 1st and JP 2nd media as that of intact ECP-T, which indicated that the tableting process did not affect the acid-resistibility of the particle. In addition, ODT $_{\mathrm{RAC}}$ rapidly disintegrated in vivo $(<30 \mathrm{~s})$, even at a high compression force (at $9 \mathrm{kN}$ ). These findings clearly suggest that RACTAB ${ }^{\circledR}$ technology is a useful approach to prepare ODTs containing enteric-coated particles.

Key words orally disintegrating tablet; enteric-coated particle; controlled release; tamsulosin hydrochloride; suspension spray-coating method

Many patients, particularly children and the elderly, often have difficulty swallowing conventional solid dosage forms such as tablets and capsules because the ability to swallow deteriorates with age. Medication compliance and therapeutic effects could be improved in these patients by taking orally disintegrating tablets (ODTs). ${ }^{1-3)}$ Many companies have developed various types of ODTs, and numerous reports have been published regarding the technologies available to prepare ODTs. ${ }^{4-15)}$ For manufacturing these ODTs, special equipments are usually required such as a freeze-dryer for wet mass filled into push-through-pack (PTP) packaging, a tabletting machine for wet mass methods, and drying and wetting chambers for the crystalline transition of amorphous sucrose. On the contrary, ODTs prepared by direct compression methods were reported to show incomplete dissolution and swelling of contained additives that resulted in a rough feeling on the tongue and delayed disintegration in the mouth. In addition, for practical use, these ODT caused a decrease in tablet hardness during storage in high-humidity conditions $\left(40^{\circ} \mathrm{C}, 75 \%\right.$ room humidity $(\mathrm{RH})$ ) because disintegrant has high hygroscopicity and causes swelling.

In our previous study, a new ODT possessing high physical strength (including clinical handling and friability) at a low compression force was successfully designed with rapidly disintegrating granules (RDGs). ${ }^{16)}$ These RDGs consisted of mannitol, the surface of which was completely coated by corn starch and crospovidone particles. Surface coating of mannitol was performed by the suspension spray-coating method.

The authors declare no conflict of interest.
These RDGs possessed a large surface area, narrow particle size distribution, and numerous micropores. Furthermore, by applying micronized ethylcellulose (MEC), the ODT acquired high resistance to humidity and high physical strength. ${ }^{17)}$ When RDGs containing MEC were tableted at an appropriate compression force $(6 \mathrm{kN})$, the ODTs exhibited adequate hardness and friability for clinical use. This newly developed technology, named RACTAB ${ }^{\circledR}$ technology, is now widely used for the marketed products of ODTs.

Recently, new types of ODTs containing functional particles have been developed based on the requests from patients to enhance their QOL. ${ }^{18-23)}$ Concerning tamsulosin hydrochloride (TAM), ODTs containing sustained-release micro-particles (under $200 \mu \mathrm{m}$ in diameter) of TAM were developed to reduce the occurrence and severity of adverse effects. ${ }^{24)}$ The entrapment of drug substances into polymer-based microspheres or microcapsules has also become an attractive approach to mask the taste of bitter drugs by keeping the drugs from coming into contact with patients' taste buds. ${ }^{19)}$ However, most ODTs containing functional particles have been prepared with a low compression force to avoid a loss-of-function during tableting, which may result in low hardness and high friability of the tablet. $^{20)}$

In this study, to improve these undesired properties of ODTs containing functional particles, RACTAB ${ }^{\circledR}$ technology is applied for the formulation design of ODTs containing enteric-coated particles. ODTs containing enteric-coated particles of TAM (ECP-T) were prepared using RACTAB ${ }^{\circledR}$ technology and their physical properties, such as compressibility (tablet hardness), friability, tablet thickness, and oral 
disintegration time were evaluated as well as the controlled release profile and the acid resistivity.

\section{Experimental}

Materials Tamsulosin hydrochloride (TAM) was obtained from Sanyo Chemical Laboratory Co., Ltd. (Toyama, Japan). D-Mannitol was purchased from Merck Co., Ltd., Japan. Corn starch (Nippon Shokuhin Kako Co., Ltd., Japan) and crospovidone (ISP Co., Ltd., Japan) were used as wicking agents and micronized ethylcellulose (Dow Chemical Co., Ltd., U.S.A.) was used as a functional additive. Crystal cellulose spheres (Celpher ${ }^{\circledR}$ CP-102: Asahi Chemical Industry Co., Ltd., Tokyo, Japan) were used as core particles, and polymethacrylate (EUDRAGIT ${ }^{\circledR}$ L30D-55 and NE30D: Evonic Degussa Japan Co., Ltd., Tokyo, Japan), ethlycellulose (EC) (Dow Chemical Co., Ltd.), hydroxylpropyl methylcellulose (HPMC) (Shin-Etsu Chemical Co., Ltd., Tokyo, Japan) and ethylcellulose dispersion (Aquacoat ${ }^{\circledR}$ ECD-30: Asahi Chemical Industry Co., Ltd.) were used as controlled release polymers for enteric-coated particles (ECP). Aspartame (Ajinomoto Healthy Supply Co.,
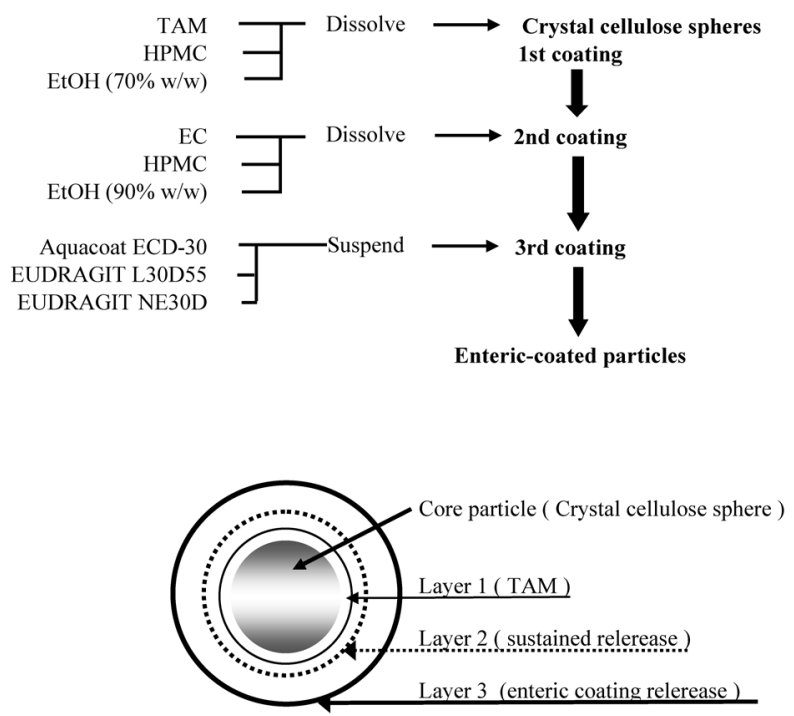

Fig. 1. Preparation Process for Enteric-Coating Particles (ECP-T) and Schematic Representations of the ECP-T Structure
Ltd., Tokyo, Japan) and Peppermint micron (Takasago Industry Co., Ltd., Tokyo, Japan) were used as sweetener and flavor, respectively. All other materials used in this study were of Japanese Pharmacopoeia (JP) grade.

Preparation of Enteric-Coated TAM Particles (ECP-T) Figure 1 shows the process used to prepare enteric-coated particles containing TAM (ECP-T) tested in this study with an illustration of the structure. ECP-T was prepared according to the method reported. ${ }^{24-26)}$ As shown in Fig. 1, ECP-T was composed of a core particle of microcrystalline cellulose, a layer of media containing TAM (Layer 1), a sustained-release membrane of water-soluble and water-insoluble polymers (Layer 2), and an enteric layer coating over the sustainedrelease membrane (Layer 3). The ratio of core particle, Layer 1,2 and 3 were $44.4,1.8,16.7$ and $37.1 \mathrm{w} / \mathrm{w} \%$ of total weight to ECP-T, respectively.

Preparation of Orally Disintegrating Tablets (ODTs) Containing ECP-T In this study, two kinds of ODTs containing ECP-T were prepared according to the formulas shown in Table 1 and Fig. 2. Figure 2a shows the preparation method of ODTs using RACTAB $^{\circledR}$ technology $\left(\right.$ ODT $_{\text {RAC }}$ ) developed in Towa Pharmaceutical Co., Ltd. ${ }^{16,17)}$ Rapidly disintegrating granules (RDGs) were initially prepared using the suspension spray-coating method with a fluidized-bed granulator (MP-01, Powrex, Japan) at a $1 \mathrm{~kg}$ size scale. Briefly, D-mannitol $\left(\mathrm{D}_{50}\right.$ : $64.3 \mu \mathrm{m})$ was spray-coated with a suspension of corn starch and crospovidone (9:1 w/w ratio). In this study, RDGs having the similar size with ECP-T (about $150 \mu \mathrm{m}$ ) were used to avoid the nonuniform distribution of ECP-T in $\mathrm{ODT}_{\mathrm{RAC}}$. The RDGs were blended with ECP-T and micronized ethylcellulose (MEC, 5\% (w/w)), and light anhydrous silicic acid and magnesium stearate were then added and shaken by hand in a plastic bag. After mixing, the resultant mixture was compressed using a rotary tableting machine (VIRG, Kikusui Seisakusho, Japan) to obtain an $\mathrm{ODT}_{\mathrm{RAC}}$ of $220 \mathrm{mg}$ in weight. The punching size and shape (curvature radius) were $\phi 8.5 \mathrm{~mm}$ and $14 \mathrm{~mm}$, respectively. Figure $2 \mathrm{~b}$ shows the preparation method of ODTs used as a reference $\left(\mathrm{ODT}_{\text {ref }}\right) . \mathrm{ODT}_{\text {ref }}$ was prepared using the direct compaction method of ECP-T, D-mannitol (SD), corn starch, crospovidone, light anhydrous silicic acid, and magnesium stearate. MEC was not included in $\mathrm{ODT}_{\text {ref }}$.

Table 1. Mean Particle Side $\left(\mathrm{D}_{50}\right)$ of Materials and Formulations of Rapidly Disintegrating Particles and Orally Disintegrating Tablets $\left(\mathrm{ODT}_{\mathrm{RAC}}\right.$, $\left.\mathrm{ODT}_{\text {ref }}: 220 \mathrm{mg} / \mathrm{table}\right)$

\begin{tabular}{|c|c|c|c|c|}
\hline \multirow{2}{*}{ Material } & \multirow{2}{*}{$(\text { Conditions })^{c}$} & \multirow{2}{*}{$\begin{array}{l}\text { Particle size } \\
\mathrm{D}_{50}(\mu \mathrm{m})\end{array}$} & \multicolumn{2}{|c|}{ Formula. No. } \\
\hline & & & $\mathrm{ODT}_{\text {ref }}$ & $\mathrm{ODT}_{\mathrm{RAC}}$ \\
\hline $\mathrm{ECP} \mathrm{T}^{a}$ & & $175.1^{d)}$ & 8 & 8 \\
\hline D-Mannitol & & $64.3^{d)}$ & - & 62 \\
\hline D-Mannitol (SD) $)^{b)}$ & & $100^{e)}$ & 67 & - \\
\hline Corn starch & (Sus.) & $19.9^{d)}$ & - & 21.6 \\
\hline Corn starch & (Extra.) & $19.9^{d)}$ & 21.6 & - \\
\hline Crospovidone & (Sus.) & $16.1^{d)}$ & - & 2.4 \\
\hline Crospovidone & (Extra.) & $16.1^{d)}$ & 2.4 & - \\
\hline Micronized ethylcellulose & (Extra.) & $5 \cdot 10^{e)}$ & - & 5 \\
\hline Light anhydrous silicic acid & (Extra.) & $3.2^{e)}$ & 0.2 & 0.2 \\
\hline Magnesium stearate & (Extra.) & $6.9^{e)}$ & 0.5 & 0.5 \\
\hline Sweetener/Flavor & (Extra.) & & 0.3 & 0.3 \\
\hline Total $(\%)$ & & & 100 & 100 \\
\hline
\end{tabular}

a) ECP-T; enteric-coating particles containing Tamsulosin hydrocholide. b) D-Mannitol (SD); spray-dried grade. c) Conditions; (Sus.); susupension, (Extra.); extra-addition. d) Measured by laser particle counter (LA-920, HORIBA). e) Cited from production catalogues. 


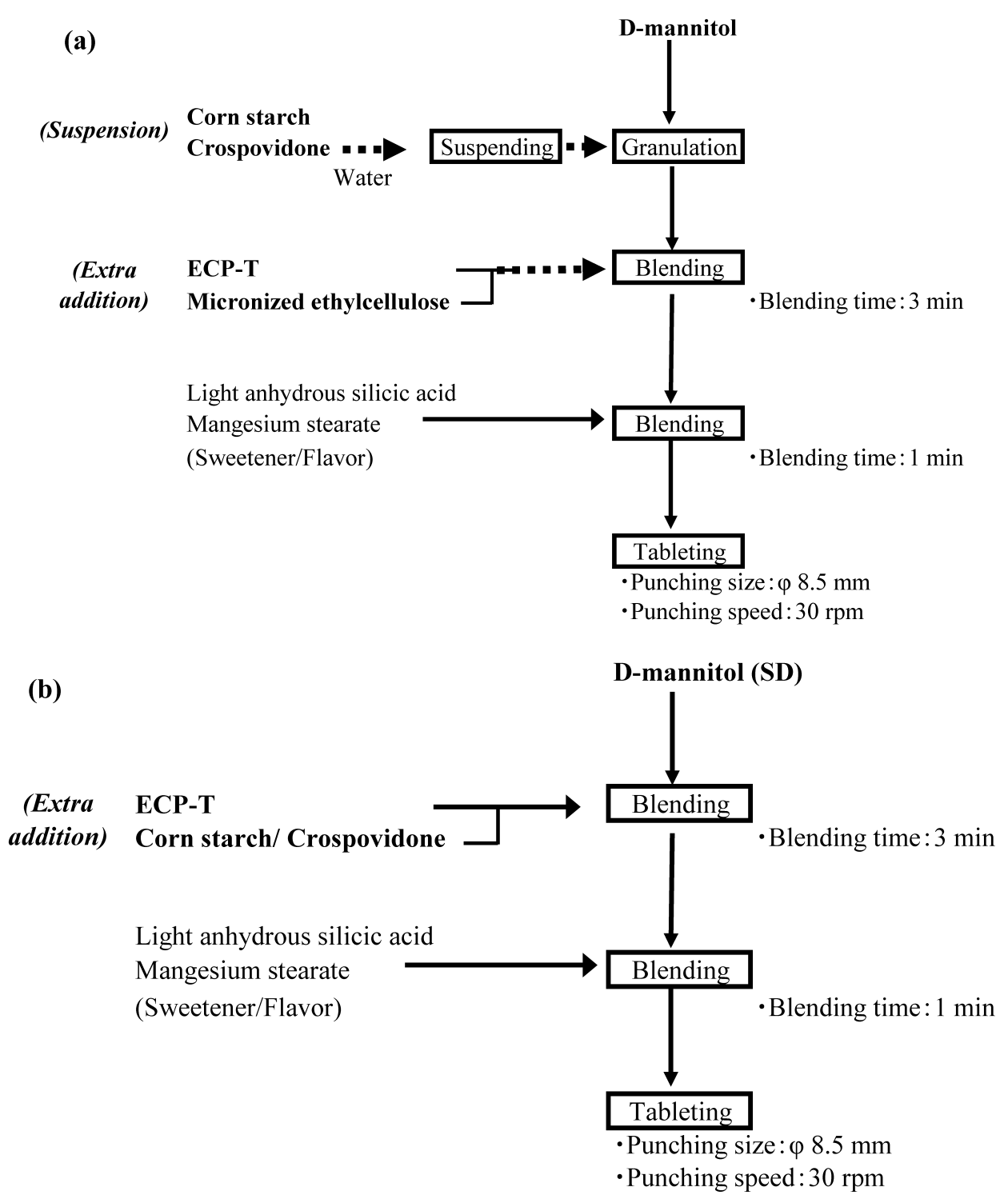

Fig. 2. (a) Manufacturing Process for Orally Disintegrating Tablets Containing ECP-T $\left(\mathrm{ODT}_{\mathrm{RAC}}\right)$ Using a Fluidized-Bed Granulator Rapidly disintegrating granules (RDGs) were prepared using the suspension spray-coating method.

(b) Manufacturing Process for Orally Disintegrating Tablets Used as a Reference $\left(\mathrm{ODT}_{\text {ref }}\right.$ ) by the Direct Compaction Method

Evaluation of the Physicochemical Properties of ECP-T and ODTs Particle Size of ECP-T: The mean particle size $\left(D_{50}\right)$ of the granules was measured using a laser particle counter (LA-920, HORIBA Corporation, Japan). Tested granules were sieved with 30 mesh (screen size of $500 \mu \mathrm{m}$ ) and measured by dry dispersion method. For dry dispersion of the tested granules, a sample amount of approximately $5 \mathrm{~g}$ was introduced into the dry powder disperser.

Scanning Electron Microscopy (SEM) of ECP-T: After the granules were sputtered with Au-Pd, SEM images of the granules were obtained using a scanning electron microscope (VE7800S; KEYENCE, Japan).

X-Ray Inspection Tomograms (CT-Scanning) of ODTs: X-Ray inspection tomograms (CT-scanning) of tablets were obtained using an X-ray CT-scan analyzer (SMX-100CT; Shimadzu, Japan). An X-ray photographing device is equipped with a pressure tube which presses the target region of ODTs.

Hardness and Thickness of ODTs: The hardness and thickness of the tablet were determined in 5 random tablets using a tablet hardness tester (TBH450 WTD IC, ERWEKA Corporation, Germany) for each formulation.

Friability of ODTs: Tablet friability was measured as the percentage weight loss of 24 tablets tumbled in a friabilator (TFF-03, TSUTSUI Scientific Instruments Corporation, Japan). The dust of tablets was removed after 4 min of rotation at $25 \mathrm{rpm}$ and the percentage weight loss was calculated.

In Vitro Dissolution Study of ODTs An in vitro dissolution study of ODT formulations was performed using JP dissolution test apparatus (Toyama Sangyo Co., Ltd., Japan). The study was conducted in $900 \mathrm{~mL}$ of JP 1st and JP 2nd fluids as the dissolution medium with a paddle speed of $50 \mathrm{rpm}$ at a temperature of $37 \pm 0.5^{\circ} \mathrm{C}$. The concentration of the drug (TAM) in the sample was analyzed using HPLC (Alliance 2487: Waters Corp., Milford, MA, U.S.A.) at a wavelength of $210 \mathrm{~nm}$. Samples were filtered with $0.2 \mu \mathrm{m}$ cellulose acetate filters, and $50 \mu \mathrm{L}$ of sample was then injected. The column used was a stainless steel column $3.0 \mathrm{~mm}$ in diameter on the inside and $50 \mathrm{~mm}$ in length, packed with octadecylsilanized 


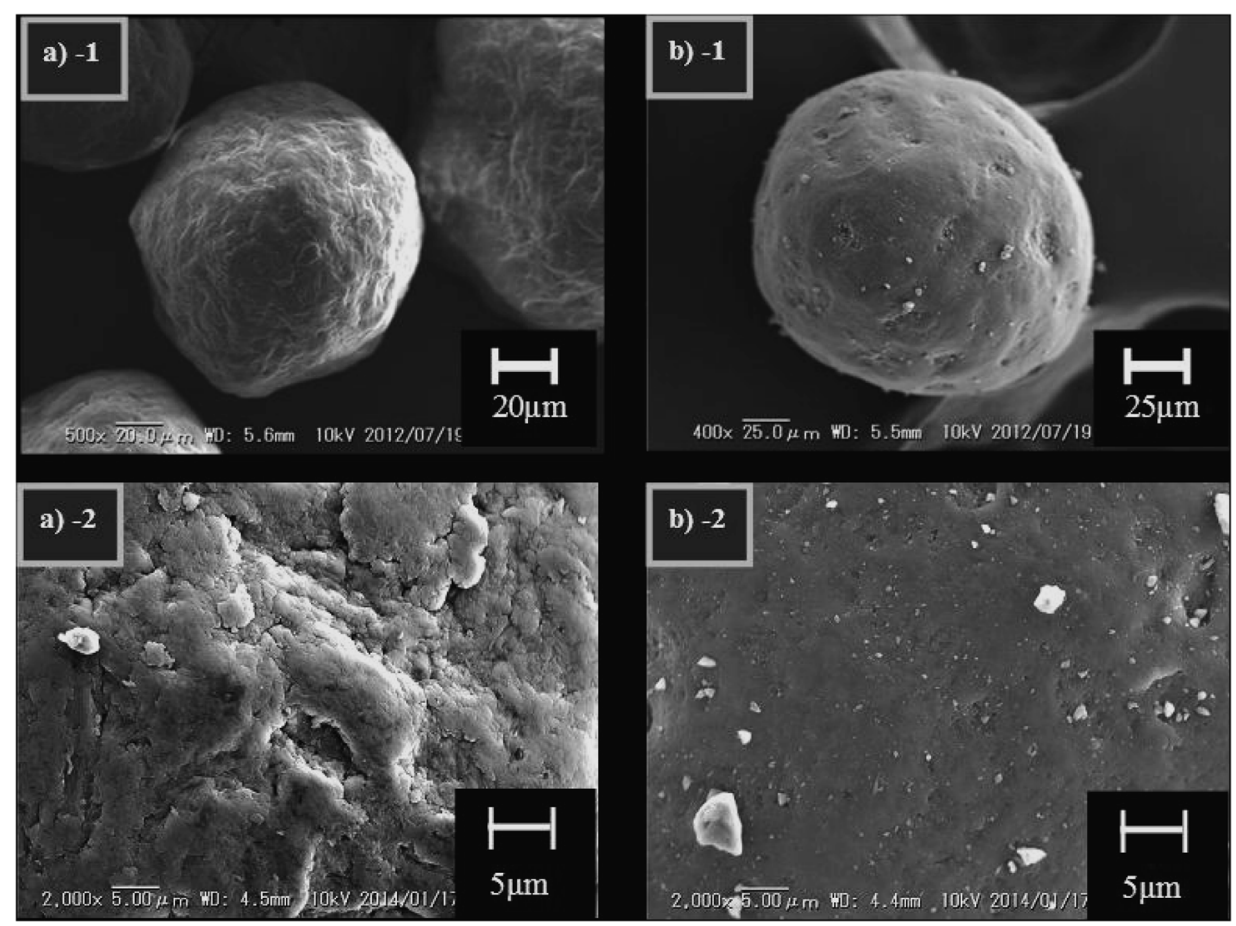

Fig. 3. Scanning Electron Micrographs of (a-1, 2) the Intact Core Particle of Microcrystalline Cellulose and (b-1, 2) Enteric-Coating Particle (ECP-T)

silica gel (Cadenza CD-C18, $3 \mu \mathrm{m}$ particle diameters, Imtakt, Japan). The mobile phase contained a mixture of diluted phosphoric acid (1 in 1000) and methanol in a volume ratio of $7: 3$. Analysis condition was isocratic elution. The flow rate of mobile phase was $0.5 \mathrm{~mL} / \mathrm{min}$. The retention time of TAM was $3 \mathrm{~min}$.

Disintegration Time of ODTs in the Oral Cavity The complete disintegration time of tablets in the oral cavity was evaluated in five healthy volunteers. The endpoint for disintegration in the mouth was the time when the tablet placed on the tongue had disintegrated until no lumps were remaining. The volunteers rinsed out their mouths with water before the test. The protocol and experimental design for all disintegration tests in the oral cavity were approved by the Ethical Committee of TOWA Pharmaceutical Co., Ltd. Five male volunteers participated in this study after informed consent of the study protocol. No adverse reactions were observed in any volunteers throughout the whole study period.

Stability of Tablet Hardness and Friability under Accelerated Conditions To assess stability under accelerated conditions, $\mathrm{ODT}_{\mathrm{RAC}}$ was stored at $40^{\circ} \mathrm{C}, 75 \% \mathrm{RH}$, open, for 1 month. After 2 weeks and 1 month, ODT $_{\text {RAC }}$ was assessed for changes in tablet hardness and friability. Hardness was determined in 5 random tablets using a tablet hardness tester. Friability was measured as the percentage weight loss of 24 tablets tumbled in a friabilator.

\section{Results}

Enteric-Coated Particles Containing TAM (ECP-T) Figure 3 shows the SEM images of the intact core particle of microcrystalline cellulose $(a-1,2)$ and ECP-T $(b-1,2)$. The particle size distribution of ECP-T is shown in Fig. 4. The mean particle size of ECP-T was $175.1 \mu \mathrm{m}$ as a $\mathrm{D}_{50}$ value (Table 1). The surface of ECP-T was smoother than that of the intact core particle because ECP-T was uniformly coated by the

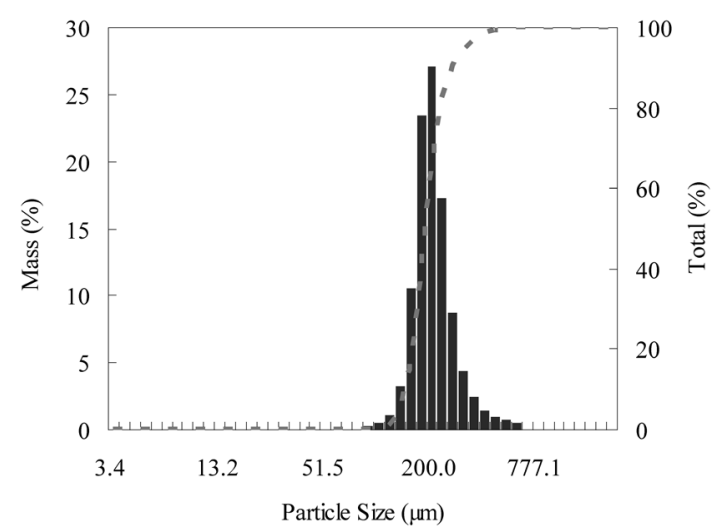

Fig. 4. Particle Size Distribution of ECP-T

dissolution-controlling membrane film, which was composed of mixtures of water-soluble and insoluble polymers.

Physical Properties of Orally Disintegrating Tablets Containing ECP-T RACTAB $^{\circledR}$ technology was used in this study to prepare functional ODTs containing ECP-T. Two different ODTs, ODT $\mathrm{RAC}_{\text {and }}$ aDT ref, were prepared to evaluate the impact of RACTAB ${ }^{\circledR}$ technology on the physical proper-

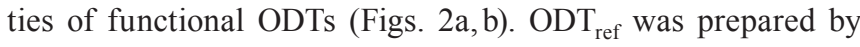
the direct compaction method without MEC.

The relationship between the compression force and tablet hardness of two ODTs is shown in Fig. 5a. Figure 5b shows the relationship between the hardness and thickness of ODTs. When a higher compression force was used, tablet hardness increased and thickness decreased in both ODTs. However, at the same compression force, $\mathrm{ODT}_{\mathrm{RAC}}$ acquired higher hardness than ODT $_{\text {ref }}$. At the same hardness, the tablet thickness of $\mathrm{ODT}_{\mathrm{RAC}}$ was also significantly greater than that of ODT $\mathrm{ref}$.

Influence of the Tableting Process on the Structure of ECP-T Figure 6 shows SEM images of intact ECP-T (a), 
(a)

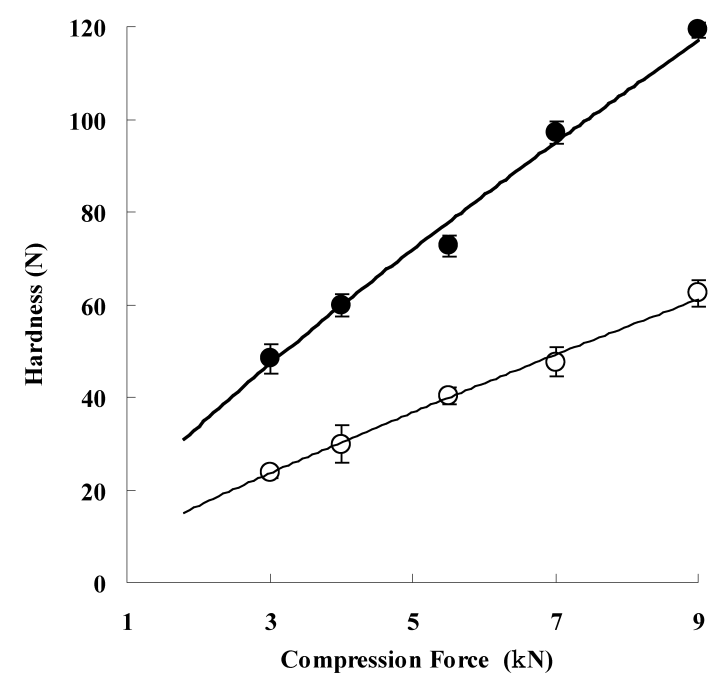

(b)

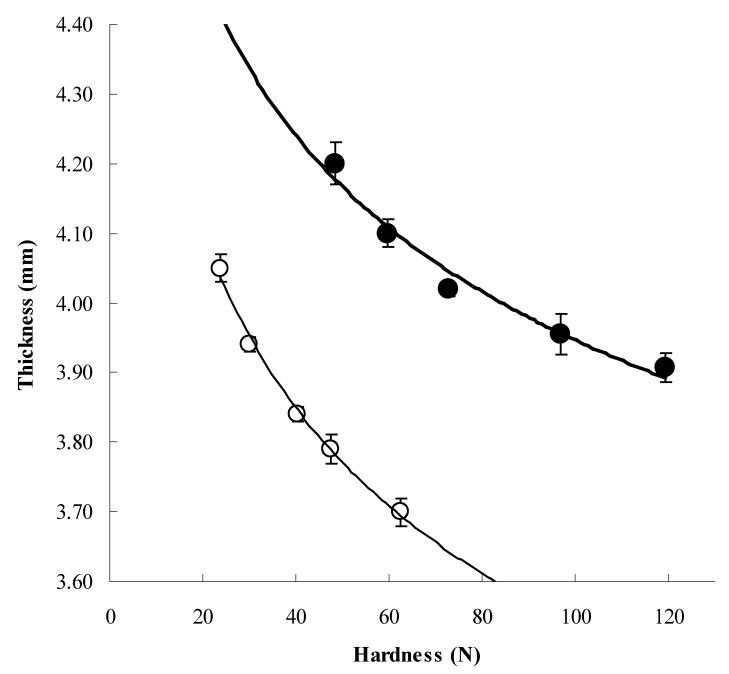

Fig. 5. (a) Relationship between Compression Force and Tablet Hardness for Various ODTs (b) Relationship between Tablet Hardness and Thickness for Various ODTs

(a) Date are expressed as mean \pm S.D. $(n=6) ; \mathrm{ODT}_{\text {ref }}(\bigcirc), \mathrm{ODT}_{\mathrm{RAC}}(\bullet)$. (b) Date are expressed as mean $\pm \mathrm{S} . \mathrm{D} .(n=6) ; \mathrm{ODT}_{\text {ref }}(\mathrm{O}), \mathrm{ODT}_{\mathrm{RAC}}(\bullet)$.
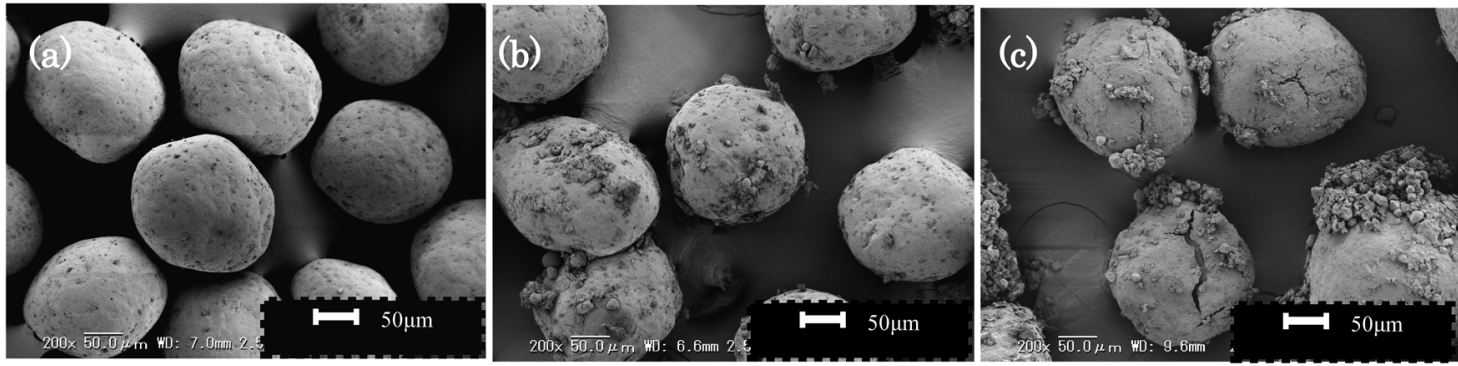

Fig. 6. Scanning Electron Micrographs of (a) Intact ECP-T, (b) ECP-T Recovered from ODT $_{\mathrm{RAC}}$, and (c) ECP-T Removed from ODT ${ }_{\text {ref }}$

ECP-T recovered from $\mathrm{ODT}_{\mathrm{RAC}}(\mathrm{b})$, and ECP-T recovered from $\mathrm{ODT}_{\text {ref }}$ (c) tableted at a compression force of $7 \mathrm{kN}$. The surface of intact ECP-T was coated smoothly with no cracks. Some adhesive substances were found on the surface of ECP-T recovered from $\mathrm{ODT}_{\mathrm{RAC}}$; however, its film layer remained intact. In contrast, the surface of ECP-T recovered from ODT was clearly damaged and had several cracks.

In Fig. 7, ECP-T in $\mathrm{ODT}_{\mathrm{RAC}}$ was observed using an X-rayCT scan analyzer. In $\mathrm{ODT}_{\mathrm{RAC}}$, ECP-T was uniformly distributed and the dissolution-controlling membrane on the surface of ECP-T remained intact as a clear film layer.

Functional Characteristics (Controlled Release Profiles) of ODTs Figure 8 shows the dissolution rate of TAM from ODTs in dissolution medium JP 1st $(\mathrm{pH}$ 1.2) and JP 2nd $(\mathrm{pH}$ 6.8). ODTs were prepared at a compression force of $7 \mathrm{kN}$. Intact ECP-T was also used as a reference. In Fig. 8a, the dissolution rate of TAM from $\mathrm{ODT}_{\mathrm{RAC}}$ at $\mathrm{pH} 1.2$ was almost the same as that from intact ECP-T, which indicated that the acidresistivity of ECP-T was not affected by the tableting process. $\mathrm{ODT}_{\mathrm{RAC}}$ and intact ECP-T also had the same dissolution profile of TAM in JP 2nd (Fig. 8b).

In contrast, the dissolution rate of TAM from $\mathrm{ODT}_{\text {ref }}$ was significantly accelerated in JP 1 st (17\% at $120 \mathrm{~min})$, suggesting the loss of acid-resistivity due to damage on ECP-T during the tableting process.

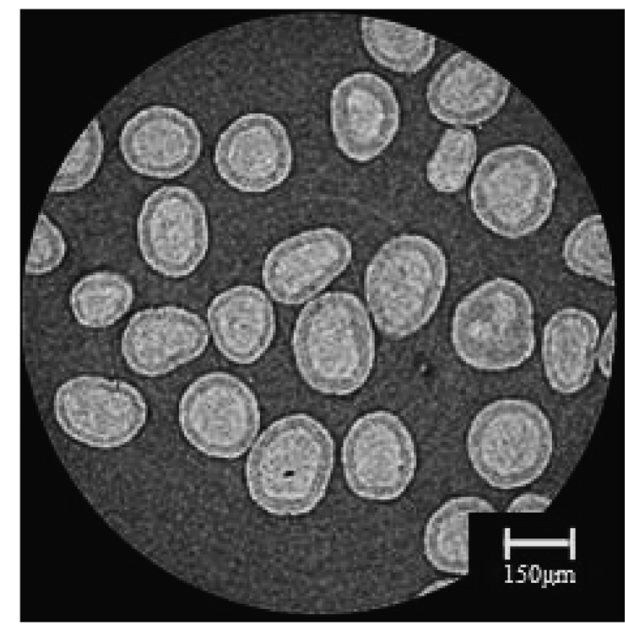

Fig. 7. X-Ray Inspection Tomograms of ECP-T in $\mathrm{ODT}_{\mathrm{RAC}}$

Figure 9 shows the effect of the compression force on the dissolution rate of TAM in JP 1st from $\mathrm{ODT}_{\mathrm{RAC}}$. The dissolution rate from $\mathrm{ODT}_{\mathrm{RAC}}$ was not influenced by tableting even at a high compression force $(9 \mathrm{kN})$.

Disintegration Time of ODTs in the Oral Cavity Figure 10 shows the relationship between the hardness of ODT $_{\text {RAC }}$ 
a) JP 1st (pH 1.2)

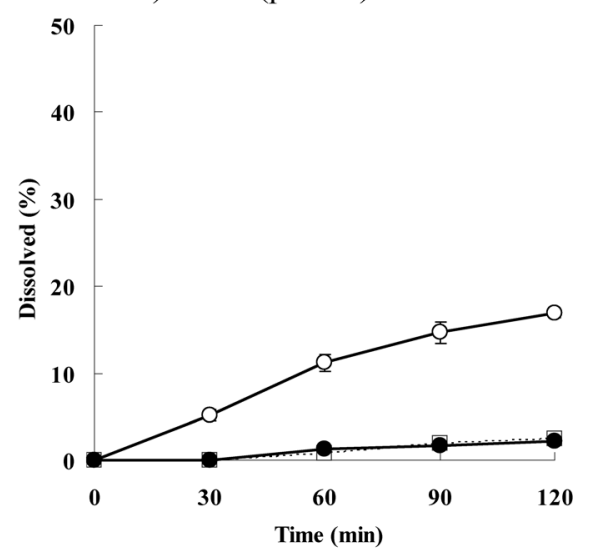

b) JP 2nd (pH 6.8)

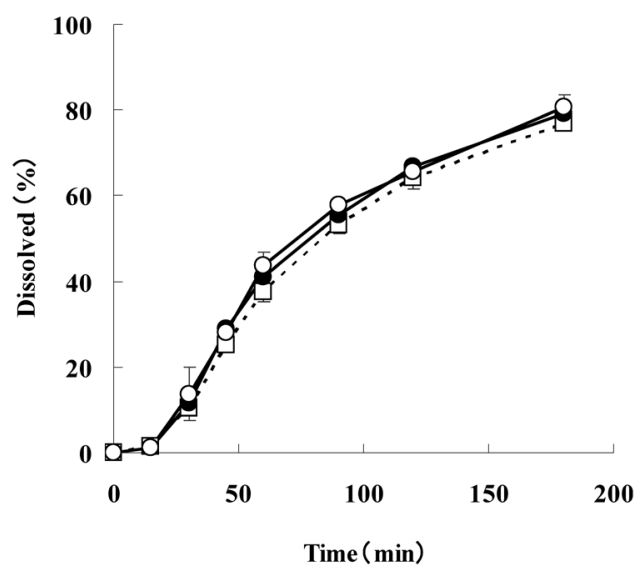

Fig. 8. Comparison of the Dissolution Profiles of TAM from Various ODTs and Enteric-Coating Particles by the Paddle Method at $50 \mathrm{rpm}$ in (a) JP 1st ( $\mathrm{pH} 1.2)$ and (b) JP 2nd (pH 6.8)

Date are expressed as mean \pm S.D. $(n=6)$; $\mathrm{ODT}_{\text {ref }}(\bigcirc), \mathrm{ODT}_{\mathrm{RAC}}(\bullet), \mathrm{ECP}-\mathrm{T}(\square)$.

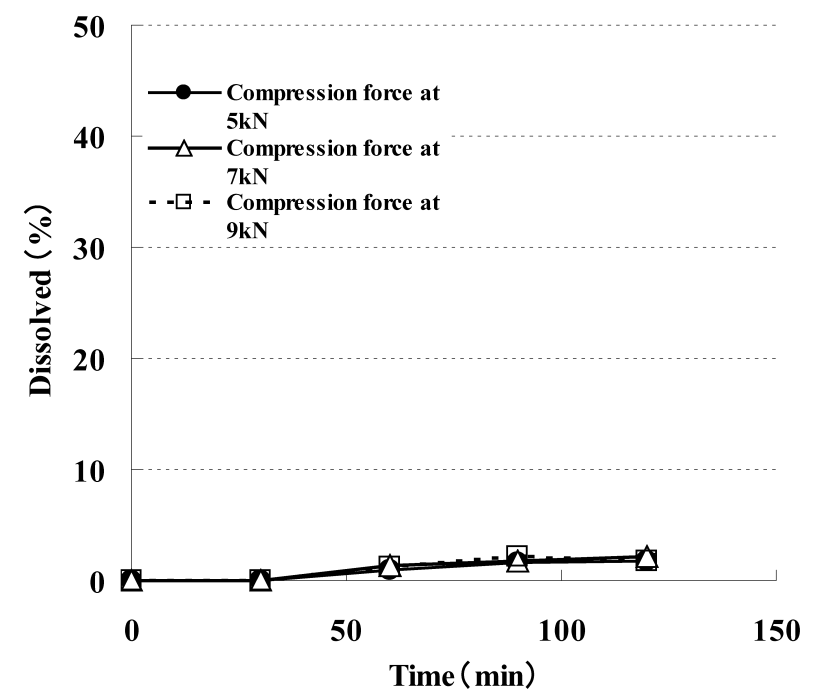

Fig. 9. Comparison of Dissolution Profiles for TAM from $\mathrm{ODT}_{\mathrm{RAC}}$ Compressed at Various Compression Forces $(5 \mathrm{kN}$ to $9 \mathrm{kN})$ by Paddle Method at $50 \mathrm{rpm}$ in (a) JP 1st ( $\mathrm{pH} 1.2)$

Date are expressed as mean \pm S.D. $(n=6) ; 5 \mathrm{kN}(\bullet), 7 \mathrm{kN}(\triangle), 9 \mathrm{kN}(\square)$.

compressed at various forces and disintegration time in the oral cavity in humans (oral disintegration time). Oral disintegration time increased linearly with the hardness of the tablet, but was shorter than $30 \mathrm{~s}$ for $\mathrm{ODT}_{\mathrm{RAC}}$ with hardness less than $100 \mathrm{~N}$. Since it is desirable for ODTs is to have a rapid disintegration time (generally less than $30 \mathrm{~s}$ ) and adequate hardness (greater than $50 \mathrm{~N}$ ), we demonstrated that $\mathrm{ODT}_{\mathrm{RAC}}$ tested in Fig. 9 satisfied these requirements as ODTs (except the hardest one with $120 \mathrm{~N})$.

Finally, the stability of the hardness, and friability of $\mathrm{ODT}_{\mathrm{RAC}}$ at a compression force of $7 \mathrm{kN}$ was evaluated under accelerated stability test conditions $\left(40^{\circ} \mathrm{C}, 75 \% \mathrm{RH}\right.$, one month for International Conference on Harmonisation of Technical Requirements for Registration of Pharmaceuticals for Human Use: ICH) (Fig. 11). The tablet hardness of $\mathrm{ODT}_{\text {RAC }}$ containing MEC (5\% (w/w)) decreased only slightly during 1 month, but was always over $50 \mathrm{~N}$. On the other hand, the friability of $\mathrm{ODT}_{\mathrm{RAC}}$ increased only slightly during 1

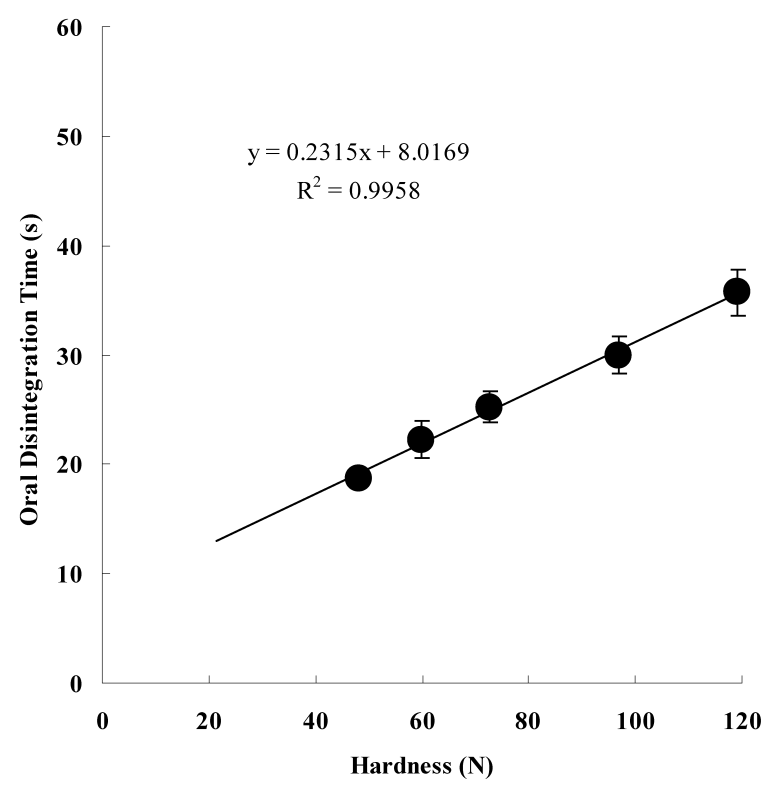

Fig. 10. Relationship between the Oral Disintegration Time of $\mathrm{ODT}_{\mathrm{RAC}}$ and Its Hardness

Date are expressed as mean \pm S.D. $(n=5)$.

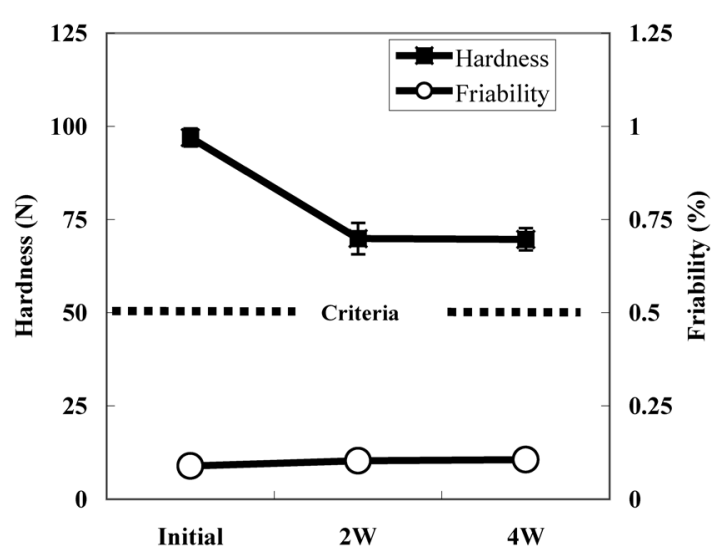

Fig. 11. Stability of Tablet Hardness and Friability under Accelerated Conditions $\left(40^{\circ} \mathrm{C}, 75 \% \mathrm{RH}\right)$ for $\mathrm{ODT}_{\mathrm{RAC}}$

Date are expressed as mean \pm S.D. $(n=6)$. 
month, but was always below $0.2 \%$.

\section{Discussion}

In this study, ODTs containing ECP-T were prepared using the RACTAB ${ }^{\circledR}$ technology $\left(\mathrm{ODT}_{\mathrm{RAC}}\right)$ and the direct compaction method $\left(\mathrm{ODT}_{\mathrm{ref}}\right)$. Then the physical properties of both ODTs were compared to investigate the applicability of RACTAB $^{\circledR}$ technology to the manufacture of ODTs containing functional particles.

Mizumoto et al. and Baldi and Malfertheriner previously reported that a particle size with an acceptable feeling in the mouth was around $150 \mu \mathrm{m}$ and $330 \mu \mathrm{m}$, respectively. ${ }^{25,27)}$ Therefore, the target size of functional particles was set to $200 \mu \mathrm{m}$ or less. In addition, we have set the criterion to guarantee the enteric-coated property of ECP-T where the dissolved amount of TAM should not exceed $10 \%$ of total amount at $120 \mathrm{~min}$ in the JP 1st dissolution medium. This criterion was taken from the guideline for bioequivalence study of enteric-coated products.

$\mathrm{ODT}_{\mathrm{RAC}}$ exhibited adequate hardness for clinical use when tableted at a $7 \mathrm{kN}$ compression force. ECP-T recovered from $\mathrm{ODT}_{\mathrm{RAC}}$ had no damage on the surface and also had the same dissolution profiles in both JP 1st and 2nd media as those of intact ECP-T. These results clearly demonstrated that ECP-T in $\mathrm{ODT}_{\mathrm{RAC}}$ maintained sufficient function as enteric-coated particles. In addition, in Fig. 7, ECP-T showed the uniform distribution in $\mathrm{ODT}_{\mathrm{RAC}}$. The similar size of RDGs with ECP-T used in this study might enable the uniform blending of both particles in the tableting process. ${ }^{28,29)}$

In contrast, the surface of ECP-T in $\mathrm{ODT}_{\text {ref }}$ was markedly damaged by tableting and had several cracks. As a result, ECP-T lost acid-resistivity even though the hardness of ODT ${ }_{\text {ref }}$ was lower than that of ODT $\mathrm{RAC}_{\text {. }}$

Spray-dried mannitol (D-mannitol (SD)) is widely used as a filler for the direct compaction of conventional tablets or ODTs because of its high fluidity, compressibility, and solubility. Since D-mannitol (SD) particles undergo plastic deformation during tableting, tablet hardness increased with an increase in the compression force and the thickness of the tablet decreased. The tableting process has generally been divided into three processes: particle rearrangement, compression, and depression. ${ }^{30)}$ Plastic deformation of granules in the tablet has been observed after these processes. ${ }^{31)}$ In the case of $\mathrm{ODT}_{\text {ref }}$, since the particle size of ECP-T was relatively large $\left(\mathrm{D}_{50}: 175.1 \mu \mathrm{m}\right)$, hardness became low $(<50 \mathrm{~N})$ even at a compression force of $7 \mathrm{kN}$ (Fig. 5). Higher compression force is required to obtain sufficient hardness for clinical use $(>50 \mathrm{~N})$, which may subsequently lead to a higher degree of plastic deformation.

In contrast, the hardness of $\mathrm{ODT}_{\mathrm{RAC}}$ became higher than $50 \mathrm{~N}$ at a low compression force $(4 \mathrm{kN})$ (Fig. 5). In addition, $\mathrm{ODT}_{\mathrm{RAC}}$ maintained adequate acid-resistivity, even at a high compression force $(9 \mathrm{kN})$ (Fig. 9). In our previous report, we demonstrated that the compaction force between RDGs was completely transmitted and related to matrix formation because MEC worked as a dry-binder in ODTs. ${ }^{17)}$ MEC increased the contact frequency between RDGs in the tablet and led to the high hardness of the tablet with a relatively low compression force. Thus, plastic deformation in $\mathrm{ODT}_{\mathrm{RAC}}$ was considered to be smaller than that in $\mathrm{ODT}_{\text {ref. }}$.

Furthermore, in the stability test under accelerated condi- tions $\left(40^{\circ} \mathrm{C}, 75 \% \mathrm{RH}\right.$, one month), the hardness and friability of $\mathrm{ODT}_{\mathrm{RAC}}$ containing MEC $(5 \%(\mathrm{w} / \mathrm{w}))$ was maintained over $50 \mathrm{~N}$ and below $0.2 \%$, respectively (Fig. 11). These results clearly demonstrate that RACTAB ${ }^{\circledR}$ technology provides various beneficial aspects to the preparation of ODTs containing functional particles, such as enteric-coated particles.

Finally, ODTs containing the controlled-release particles of TAM prepared by RACTAB $^{\circledR}$ technology (Tamslon ${ }^{\circledR}$-D) was shown to be bioequivalent with the reference ODT $\left(\right.$ Harnal $^{\circledR}$-D). ${ }^{32)}$ This result also confirmed that it is possible to manufacture bioequivalent products having various advantages for clinical use with RACTAB ${ }^{\circledR}$ technology.

\section{Conclusion}

This study successfully demonstrated that RACTAB ${ }^{\circledR}$ technology can facilitate the future application and formulation design of ODTs. ODTs containing functional particles with high tablet hardness, high physical stability, high resistivity against humidity, and rapid disintegration in the oral cavity could be prepared by tableting the physical mixture of RDGs and MEC at an appropriate compression force. RACTAB ${ }^{\circledR}$ technology is expected to be applied to the manufacture of ODTs for a wide range of functional particles such as tastemasking of bitter drugs and sustained or controlled release.

Acknowledgments The authors wish to thank Dr. N. Oku, School of Pharmaceutical Sciences, University of Shizuoka, for many helpful suggestions.

\section{References}

1) CDER, "Dosage Forms, Data Standards Manual," 1992.

2) William R. P., Tapash K. G., "Pharmaceutical Technology.": 〈http://www.pharmtech.com/pharmtech/article/articleDetail.jsp?id= 185957), cited in 2005.

3) FDA, "Guidance for Industry.": 〈http://www.fda.gov/downloads/ Drugs/.../Guidances/ucm070578.pdf), cited in 2008.

4) Watanabe Y., Koizumi K., Zama Y., Kiriyama M., Matsumoto Y., Matsumoto M., Biol. Pharm. Bull., 18, 1308-1310 (1995).

5) Bi Y., Sunada H., Yonezawa Y., Danjo K., Otsuka A., Iida K., Chem. Pharm. Bull., 44, 2121-2127 (1996).

6) Bi Y. X., Sunada H., Yonezawa Y., Danjo K., Drug Dev. Ind. Pharm., 25, 571-581 (1999).

7) Bi Y., Yonezawa Y., Sunada H., J. Pharm. Sci., 88, 1004-1010 (1999).

8) Chang R. K., Guo X., Burnside B. A., Couch R. A., Pharm. Technol., 6, 52-58 (2000).

9) Ishikawa T., Mukai B., Shiraishi S., Utoguchi N., Fujii M., Matsumoto M., Watanabe Y., Chem. Pharm. Bull., 49, 134-139 (2001).

10) Sugimoto M., Matsubara K., Koida Y., Kobayashi M., Dev. Tech., 6, 487-493 (2001)

11) Sugimoto M., Maejima T., Narisawa S., Matsubara K., Yoshino H., Int. J. Pharm., 296, 64-72 (2005).

12) Sugimoto M., Narisawa S., Matsubara K., Yoshino H., Nakano M. Handa T., Chem. Pharm. Bull., 54, 175-180 (2006).

13) Sugimoto M., Narisawa S., Matsubara K., Yoshino H., Nakano M., Handa T., Int. J. Pharm., 320, 71-78 (2006).

14) Schiermeier S., Schmidt P. C., Eur. J. Pharm. Sci., 15, 295-305 (2002).

15) Mizumoto T., Masuda Y., Yamamoto T., Yonemochi E., Terada K., Int. J. Pharm., 306, 83-90 (2005).

16) Okuda Y., Irisawa Y., Okimoto K., Osawa T., Yamashita S., Int. J. Pharm., 382, 80-87 (2009).

17) Okuda Y., Irisawa Y., Okimoto K., Osawa T., Yamashita S., Int. J. 
Pharm., 423, 351-359 (2012).

18) Xu J., Bovet L. L., Zhao K., Int. J. Pharm., 359, 63-69 (2008).

19) Yan Y. D., Woo J. S., Kang J. H., Yong C. S., Choi H. G., Biol. Pharm. Bull., 33, 1364-1370 (2010).

20) Kondo K., Niwa T., Ozeki Y., Ando M., Danjo K., Chem. Pharm. Bull., 59, 1214-1220 (2011).

21) Aulton M. E., Dyer A. M., Khan K. A., Ind. Pharm., 20, 3069-3104 (1994).

22) Debunne A., Vervaet C., Mangelings D., Remon J. P., Eur. J. Pharm. Sci., 22, 305-314 (2004).

23) Maganti L., Celik M., Int. J. Pharm., 103, 55-67 (1994).

24) Maeda A., Shinoda T., Ito N., Baba K., Oku N., Mizumoto T., Int. J. Pharm., 408, 84-90 (2011).
25) Mizumoto T., MEMBRANE, 33, 82-84 (2008).

26) Shinoda T., Maeda A., Itou N., Mizumoto T., Yamazaki S., Takaishi Y., Patent Application WO2003/009831 (2003).

27) Baldi F., Malfertheriner P., Digestion, 67, 1-5 (2003).

28) Crooks M. J., Ho R., Powder Technol., 14, 161-167 (1976).

29) Tinke A. P., Vanhoutte K., Vanhoutte F., De Smet M., De Winter H., Int. J. Pharm., 297, 80-88 (2005).

30) Otsuka M., Hasegawa H., Matsuda Y., Chem. Pharm. Bull., 45, 894-898 (1997).

31) Shu T., Suzuki H., Hironaka K., Ito K., Chem. Pharm. Bull., 50, 193-198 (2002)

32) Toh Y., Hashizume Y., Irisawa Y., Okamoto Y., Japanese Journal of Medicine and Pharmaceutical Science, 63, 247-272 (2010). 